\title{
Fibromodulin - A New Target of Osteoarthritis Management?
}

\author{
Chenshuang $\mathrm{Li}^{1,2,3,4}$, Pin $\mathrm{Ha}^{3}$, Wenlu Jiang ${ }^{3}$, Christos S. Haveles ${ }^{5}$, Zhong Zheng ${ }^{3 *}$ \\ and Min Zou ${ }^{1,2 *}$ \\ ${ }^{1}$ Key Laboratory of Shaanxi Province for Craniofacial Precision Medicine Research, College of Stomatology, Xi'an Jiaotong \\ University, Xi'an, China, ${ }^{2}$ Department of Orthodontics, College of Stomatology, Xi'an Jiaotong University, Xi'an, China, ${ }^{3}$ Division of \\ Growth and Development, Section of Orthodontics, School of Dentistry, University of California, Los Angeles, Los Angeles, CA, \\ United States, ${ }^{4}$ Department of Orthodontics, School of Dental Medicine, University of Pennsylvania, Philadelphia, PA, United \\ States, ${ }^{5}$ David Geffen School of Medicine at University of California, Los Angeles, Los Angeles, CA, United States
}

Keywords: fibromodulin, arthritis, osteoarthritis, collagen, inflammation, keratan sulfate

\section{INTRODUCTION}

As a leading cause of disability among adults, osteoarthritis $(\mathrm{OA})$ leads to serious public health and economic burdens. Currently, treatment options for OA are generally based on symptom severity and duration, with the goals of symptom alleviation and improvement in functional status (Taruc-Uy and Lynch, 2013). Nonpharmacologic and pharmacologic strategies are used initially, while a surgical approach to OA is reserved for chronic cases when these treatments failed. Unfortunately, the currently available clinical pharmacologic treatments for OA, such as analgesia, glucocorticoids, non-steroidal anti-inflammatory drugs, and disease-modifying antirheumatic drugs, are not adequately effective (Chevalier et al., 2009; Scott, 2010; Verbruggen et al., 2012; Chevalier et al., 2015; Appleton, 2018; Li and Zheng, 2018; Li et al., 2018), and generally associated with a diversity of adverse side-effects (Habib et al., 2010; Cooper et al., 2016; Compston, 2018). For instance, analgesia does not reduce inflammation and cartilage damage (Appleton, 2018), glucocorticoids have been reported to induce severe damages in the musculoskeletal, cardiovascular, and gastrointestinal systems (Cooper et al., 2016; Compston, 2018), and non-steroidal anti-inflammatory drugs do not actively control arthritis progression (Appleton, 2018). Accumulating evidence demonstrates that an ideal OA-combating agent should be able to reduce inflammation and promote cartilage regeneration safely, which has long been desired. In responding to this demand, the current strategy for disease-modifying osteoarthritis drug seeking has shifted to biologoical molecules that promote chondorgenic development and regenration.

To date, a diversity of well-known pro-chondrogenic growth factors, such as bone morphogenetic proteins (BMPs) and transforming growth factors (TGFs), have been examined for OA treatment. However, the results are not optimistic since intra-articular injection of these growth factors could even enhance the inflammatory infiltration in damaged joints (Allen et al., 1990; Fava et al., 1991; Hong et al., 2009). Meanwhile, multiple transcriptional factors that potentially suppress inflammation, such as nuclear factor of activated T cells 1 (NFATc1), NFATc2, and runt-related transcription factor 1 (RUNX1), have also be introduced in this arena against OA, while they do not hold much promise presently. For example, the function of NFATc proteins in arthritis is controversial (Yaykasli et al., 2009; Miclea et al., 2011; Greenblatt et al., 2013).

Another possibility for fighting OA is utilizing the extracellular matrix (ECM) molecules that naturally distribute in the articular cartilage. For example, fibromodulin (FMOD) is an ECM protein with multiple keratin sulfate side-chains that belongs to the small leucine-rich proteoglycan family (Plaas et al., 1990). It was first identified as a collagen-binding molecule broadly distributed in connective tissues, with particularly high expression in cartilage (Hedbom and Heinegard, 1989). In the past three 
decades, in addition to the broad attention of its effects on collagen fibrillogenesis (Chen et al., 2010), muscle development (Lee et al., 2018a; Lee et al., 2018b), cell reprogramming (Zheng et al., 2012; Li et al., 2016; Zheng et al., 2019), angiogenesis (Jian et al., 2013; Zheng et al., 2014; Ao et al., 2017), wound healing (Zheng et al., 2017), and tumorigenesis (Pourhanifeh et al., 2019), the involvement of FMOD in cartilage development and maintenance as well as arthritis progression, especially in temporomandibular joint (TMJ) OA, has been investigated through world-wide collaboration. Here, we review the current research investigating FMOD and arthritis, and aim to provide novel insight into the potential use of FMOD for OA management.

\section{SPATIOTEMPORAL DISTRIBUTION OF FMOD DURING CARTILAGE GROWTH AND DEVELOPMENT}

An investigation that focused on mouse glenohumeral joints demonstrated that, at 12-13 days post-coitus when the limb buds are just condensing mesenchymal cells, FMOD was not detectable at the protein level (Murphy et al., 1999). Intense FMOD staining was first noticed at the surface of the scapular and humeral anlage intracellularly and pericellularly in the interzone at 14-15 days post-coitus (Murphy et al., 1999). Starting from 17 days post-coitus, a strong FMOD signal was found in the ECM surrounding the chondrocytes at the surface of the joints and proliferating chondrocytes in the epiphyses of the humerus and scapula (Murphy et al., 1999). Meanwhile, during postnatal maturation until adulthood, FMOD was detected throughout the ECM of the developing articular surface and the growth plate but was more abundant in articular cartilage (Murphy et al., 1999). Since FMOD was associated with prechondrocytic mesenchymal cells in the interzone before joint cavitation and with developing articular chondrocytes in the maturing and young adult limbs, it has been proposed that FMOD may function in the early genesis of articular cartilage (Murphy et al., 1999).

It is worth noting that FMOD shared a similar temporospatial transcriptional pattern with type II collagen in mouse knee joints during postnatal development, while FMOD gene expression reached the maximum level at 1 month old (Saamanen et al., 2001). FMOD transcription was restricted to chondrocytes and peaked in the proliferating zone and the early articular cartilage (Saamanen et al., 2001), which had been confirmed at the protein level by immunostaining (Murphy et al., 1999). In mature animals, in situ hybridization revealed that both pericellular and interterritorial cartilage at knee joints had high FMOD expression with the highest intensity in the middle and deep zones of the uncalcified cartilage (Saamanen et al., 2001). At 6 months old, FMOD staining decreased in the uncalcified cartilage but increased in the calcified cartilage (Saamanen et al., 2001). FMOD was also detected in the hypertrophic chondrocytes of the secondary ossification centers and growth plate of mice at 10 days old, and transcription of FMOD was diminished and finally disappeared with maturation and aging of the trabecular epiphyses (Saamanen et al., 2001).

\section{STRUCTURAL ALTERATION OF FMOD IN AGING AND ARTHRITIS PROGRESSION}

In addition to its spatiotemporal distribution, FMOD's structural heterogeneity was also noticed during articular cartilage growth and development. For instance, FMOD isolated from young articular cartilage carries neither $\alpha(2-6)$ linked $N$-acetylneuraminic acid nor $\alpha(1-3)$-linked fucose in the $\mathrm{N}$-linked keratan sulfate chains (Lauder et al., 1996). Meanwhile, an age-related increase has been observed in the abundance of both a(2-6)-linked $N$-acetylneuraminic acid and a(1-3)-linked fucose, but not the levels of galactose sulfation (Lauder et al., 1998). Western blot showed FMOD-derived from fetal and neonatal articular cartilage (f/n-FMOD) as a diffused region with a relative molecular weight of $70-110 \mathrm{kDa}$ (Cs-Szabo et al., 1995; Roughley et al., 1996), while FMOD-derived from mature adult (a-FMOD) was a more discrete component with a relative molecular weight of $67 \mathrm{kDa}$ (Cs-Szabo et al., 1995; Roughley et al., 1996) - larger than the FMOD core protein without posttranslational modifications $(46 \mathrm{kDa})$. Interestingly, digesting f/n-FMOD with keratanase II or endo $\beta$-galactosidase reduces its molecular weight to a similar level of a-FMOD (Cs-Szabo et al., 1995). Thus, Roughley et al. argued that FMOD might predominantly exist in the proteoglycan form in juvenile cartilage tissues but is mainly in a glycoprotein form in the adult counterparts (Roughley et al., 1996).

Interestingly, FMOD is one of the small leucine-rich proteoglycans with the most significantly increased protein fragmentation in arthritis compared with macroscopically healthy articular cartilage from the age-matched donors (Melrose et al., 2008). In addition to the $59 \mathrm{kDa}$ band, multiple small bands can be detected by Western blot when FMOD is isolated from articular cartilage of OA and rheumatoid arthritis patients (Cs-Szabo et al., 1995; Roughley et al., 1996; Melrose et al., 2008; Shu et al., 2019). Moreover, when using N-glycosidase to remove the sulfate chains from FMOD isolated from arthritic articular cartilage, several protein bands with the size of 43, 40, and $27 \mathrm{kDa}$ were detected (Cs-Szabo et al., 1995). Therefore, arthritis progression may not only alter the degree and type of its carbohydrate substation but also lead to the breakage of the FMOD core protein.

Meanwhile, degradation of FMOD core protein was also observed in interleukin (IL)-1-challenged cartilage (Sztrolovics et al., 1999; Shu et al., 2019) — a representative model that elucidates the genetic and molecular pathogenesis of inflammation-related secondary OA (Kuyinu et al., 2016). The degradation of FMOD core protein was predominantly catalyzed by matrix metalloproteinases (MMPs) and ADAM metallopeptidases with thrombospondin type 1 motifs (ADAMTSs) (Kashiwagi et al., 2004; Shu et al., 2019). In vitro digestion of healthy human knee cartilage with MMP-13, ADAMTS-4, and ADAMTS-5 generated FMOD fragments of similar sizes as FMOD derived from OA cartilage without digestion (Shu et al., 2019). Notably, the fragmented FMOD is always detected by the antibody recognizing the N-terminal fragment of FMOD but not the one recognizing the C-terminal (Melrose et al., 2008; Shu et al., 2019). One possible explanation is that the C-terminus 
is vulnerable to the fragmentation and not stably retained in the tissue, and substantially lost into the synovial fluid (Melrose et al., 2008). Importantly, MMP-13 degradation of FMOD resulted in a fragment of $30 \mathrm{kDa}$, which was also detected in moderately and severely fibrillated cartilage, instead of healthy or slightly fibrillated cartilage (Monfort et al., 2006). These phenomena may support the hypothesis that the sensitivity of FMOD protein fragmentation is increased along with the severity of cartilage degradation.

\section{LESSONS FROM FMOD DEFICIENT MICE FOR OA INVESTIGATION}

FMOD-null $\left(\mathrm{Fmod}^{-/-}\right)$mice have distinct knee joints in comparison with their wildtype (WT) littermates at 36 weeks old (Gill et al., 2002), accompanied by a significantly higher histological arthritis score (Ameye et al., 2002). In addition, serial sections through FMOD-null mice knees showed degeneration and joint remodeling histologically. More severe incidences of degeneration occurred in the area of the tibial condyles that are uncovered by the menisci, as these sites experience the highest loading stress, resulting in considerable loss of cartilage and bone thickness (Gill et al., 2002). Moreover, the menisci of FMOD-null mice had a markedly less sharp profile with more rounded edges, similar to FMOD-null ligaments, which were also more likely to be damaged compared to WT ligaments. The area of tibial articular cartilage was even more exposed due to degenerated menisci compared to that of the WT littermates (Gill et al., 2002). Furthermore, knee joints of $\mathrm{Fmod}^{-/-}$mice at 80 weeks old displayed full-depth lesions of articular cartilage and clusters of cells that were not seen in the knee joints of WT littermates (Gill et al., 2002).

As biglycan (BGN) and FMOD have overlapping and possible compensatory functions in the joints (Shirakura et al., 2017), $B G N$ and FMOD double-knockout $\left(\mathrm{Bgn}^{-/ 0} / \mathrm{Fmod}^{-/-}\right)$mice exhibit an earlier onset of OA than $\mathrm{Fmod}^{-/-}$mice. $\mathrm{Bgn}^{-/ 0} / \mathrm{Fmod}^{-/-}$mice presented with an abnormal gait characterized by the decreased flexibility of knee and ankle joints (dragging leg), which was observed as early as 3 weeks old. Additionally, at 3 months old, the histological arthritis score of the $\mathrm{Bgn}^{-/ 0} / \mathrm{Fmod}^{-/-}$knee joints was significantly higher than that of the WT knee joints. However, the abnormal gait phenomena were observed in neither $B G N$ nor FMOD single knockout mice (Ameye et al., 2002).

Moreover, BGN and FMOD are also highly expressed in the disc and articular cartilage of the TMJ (Wadhwa et al., 2005a). $\mathrm{Bgn}^{-10} / \mathrm{Fmod}^{-/-}$mice developed accelerated OA accompanied by small vertical clefts in the condylar cartilage and partial disruption of the disc as compared to WT animals at 6 months old (Wadhwa et al., 2005b). At 18 months old, extensive cartilage erosion was visible in the $\mathrm{Bgn}^{-/ 0} / \mathrm{Fmod}^{-/-}$mice TMJ (Wadhwa et al., 2005b).

\section{POTENTIAL ROLES OF FMOD IN ARTHRITIS}

There are several hypotheses about the possible roles of FMOD in arthritis. FMOD binds to collagens (Melching and Roughley, 1999), and fragmentation of FMOD during arthritis progression may destabilize collagen fibrils, rendering them more susceptible to tissue collagenases (Kashiwagi et al., 2004). However, such a difference between WT and FMOD-null mice may not necessarily have immediately visible effects at the ultrastructural level in adults (Ameye et al., 2002).

Alternatively, FMOD may sequester TGF- $\beta$ /BMP superfamily members in the ECM and thereby prevent their binding to the cellular receptors (Wadhwa et al., 2005a). For example, when treating the TMJ with BMP2, both catabolic and anabolic markers were more profoundly upregulated in the $\mathrm{Bgn}^{-10} /$ Fmod $^{-1-}$ mice than WT animals (Shirakura et al., 2017). This observation suggests that BGN and FMOD could protect the condyle from BMP2-induced matric turnover (Shirakura et al., 2017). Additionally, the sequestration of TGF- $\beta 1$ in mandibular condylar chondrocyte ECM decreased in $\mathrm{Bgn}^{-10} / \mathrm{Fmod}^{-/-}$mice. The overactive TGF- $\beta 1$ signal transduction in $\mathrm{Bgn}^{-/ 0} / \mathrm{Fmod}^{-/-}$ mice accelerated both production and degradation of type II collagen and aggrecan, and subsequently led to an overall imbalance in ECM turnover that favors cartilage degradation and the onset of OA (Embree et al., 2010).

FMOD may also function as a barrier preventing cell adhesion and subsequent cartilage damage. For example, FMOD administration dramatically prevents the adhesion of polymorphonuclear neutrophils and fibroblasts on articular cartilage surfaces (Noyori and Jasin, 1994; Mitani et al., 2001). This inhibition of cellular attachment may be attributed to the capability of FMOD to mask epitopes of cartilage collagen that face the joint cavity (Noyori and Jasin, 1994).

Furthermore, FMOD may participate in arthritis progression by directly manipulating inflammatory reactions. For instance, $\mathrm{Clq}$ and complement inhibitor factor $\mathrm{H}$ can directly bind to FMOD but in different regions (Akimoto et al., 2006). However, the deposition of the membrane attack complex and C5a release were lower in the presence of FMOD, presumably due to the formation of the FMOD-factor $\mathrm{H}$ complex (Akimoto et al., 2006). Interestingly, IL-1 only stimulates the binding of C1q, but not factor $\mathrm{H}$, to the $\mathrm{N}$-terminal fragment of FMOD in cartilage (Akimoto et al., 2006). Thus, FMOD may balance the activation of the classical complement pathway: when maintained in its intact form, FMOD silences the complement cascade by binding factor $\mathrm{H}$; on the other hand, when FMOD is degraded or fragmented, as seen in OA (Melrose et al., 2008; Shu et al., 2019), the N-terminal FMOD segment binds to C1q and in turn activates the complement system to eliminate pathogens and damaged cells for tissue recovery and reconstruction.

FMOD has been used as an early marker of chondrogenesis (Barry et al., 2001). The expression level of FMOD is inversely correlated with the passage number of human chondrocytes in monolayer cultivation (Lin et al., 2008). In the TMJ cartilage of 3-month-old $\mathrm{Bgn}^{-/ 0} / \mathrm{Fmod}^{-/-}$mice, fewer proliferative chondrocytes were noticed in comparison to that of their WT counterparts (Wadhwa et al., 2005b). Moreover, Bgn ${ }^{-10}$ / Fmod $^{-1-}$ mice presented with more chondrocyte apoptosis in the articular cartilage than WT mice at the same developmental stage (Wadhwa et al., 2005a). A recent study even showed that microRNA-340-5p negatively regulated OA chondrocyte proliferation while stimulating apoptosis by reducing FMOD 
expression (Zhang et al., 2018). Nevertheless, the exact function of FMOD in chondrogenesis has yet to be fully uncovered.

\section{FURTHER DIRECTION}

As aforementioned, FMOD is a critical ECM component involved in articular cartilage development, growth, aging, and arthritis; however, the exact functions of FMOD during arthritis are still unclear. Take advantage of the development of the Cre/Lox as well as CRISPR-Cas9 recombination system, the specific functions of FMOD during arthritis progression could be deciphered in detail with tissue-specific knockout animal models. Recently, it has been reported that FMOD can be successfully produced and purified from the cell culture supernatant of stable recombinant $\mathrm{CHO}-\mathrm{K} 1$ cells transfected with a plasmid harboring the human FMOD gene (Zheng et al., 2012; Li et al., 2016; Pourhanifeh et al.,

\section{REFERENCES}

Akimoto, T., Nonaka, T., Kitamoto, Y., Ishikawa, H., Ninomiya, H., Chikamatsu, K., et al. (2006). Radiation therapy for T2N0 laryngeal cancer: a retrospective analysis for the impact of concurrent chemotherapy on local control. Int. J. Radiat. oncology biology Phys. 64 (4), 995-1001. doi: 10.1016/j. ijrobp.2005.10.003

Allen, J. B., Manthey, C. L., Hand, A. R., Ohura, K., Ellingsworth, L., and Wahl, S. M. (1990). Rapid Onset Synovial inflammation and hyperplasia induced by transforming growth factor beta. J. Exp. Med. 171 (1), 231-247. doi: 10.1084/ jem.171.1.231

Ameye, L., Aria, D., Jepsen, K., Oldberg, A., Xu, T., and Young, M. F. (2002). Abnormal collagen fibrils in tendons of biglycan/fibromodulin-deficient mice lead to gait impairment, ectopic ossification, and osteoarthritis. FASEB J. 16 (7), 673-680. doi: 10.1096/f.01-0848com

Ao, Z., Yu, S., Qian, P., Gao, W., Guo, R., Dong, X., et al. (2017). Tumor angiogenesis of SCLC inhibited by decreased expression of FMOD via downregulating angiogenic factors of endothelial cells. Biomedicine \& Pharmacotherapy 87, 539-547. doi: 10.1016/j.biopha.2016.12.110

Appleton, C. T. (2018). Osteoarthritis year in review 2017: biology. Osteoarthritis Cartilage 26 (3), 296-303. doi: 10.1016/j.joca.2017.10.008

Barry, F., Boynton, R. E., Liu, B., and Murphy, J. M. (2001). Chondrogenic differentiation of mesenchymal stem cells from bone marrow: differentiationdependent gene expression of matrix components. Exp. Cell Res. 268 (2), 189200. doi: 10.1006/excr.20015278

Chen, S., Oldberg, A., Chakravarti, S., and Birk, D. E. (2010). Fibromodulin regulates collagen fibrillogenesis during peripheral corneal development. Dev. Dynamics : An Off. Publ. Am. Assoc. Anatomists 239 (3), 844-854. doi: 10.1002/ dvdy. 22216

Chevalier, X., Goupille, P., Beaulieu, A. D., Burch, F. X., Bensen, W. G., Conrozier, T., et al. (2009). Intraarticular injection of Anakinra in osteoarthritis of the Knee: a multicenter, randomized, double-blind, placebo-controlled study. Arthrit Rheum-Arthr 61 (3), 344-352. doi: 10.1002/art.24096

Chevalier, X., Ravaud, P., Maheu, E., Baron, G., Rialland, A., Vergnaud, P., et al. (2015). Adalimumab in patients with hand osteoarthritis refractory to analgesics and NSAIDs: a randomised, multicentre, double- blind, placebo-controlled trial. Ann. Rheum Dis. 74 (9), 1697-1705. doi: 10.1136/ annrheumdis-2014-205348

Compston, J. (2018). Glucocorticoid-induced osteoporosis: an update. Endocrine 61 (1), 7-16. doi: 10.1007/s12020-018-1588-2

Cooper, C., Bardin, T., Brandi, M. L., Cacoub, P., Caminis, J., Civitelli, R., et al. (2016). Balancing benefits and risks of glucocorticoids in rheumatic diseases and other inflammatory joint disorders: new insights from emerging data. An expert consensus paper from the European Society for Clinical and Economic
2019). Since FMOD whole protein is now easy to produce, further in-depth investigations are warranted to reveal the underlying mechanism of action of FMOD as a new generation disease-modifying osteoarthritis drug candidate. Last but not least, the plasmid- or virus-mediated expression, as well as directly synthesis, could be utilized to identify the functional sequence(s) of FMOD that regulate(s) cartilage development and pathology, which would further advance the pharmacology application of FMOD.

\section{AUTHOR CONTRIBUTIONS}

CL conceived the opinion. $\mathrm{CL}, \mathrm{PH}$, and $\mathrm{ZZ}$ wrote and revised the manuscript. WJ and $\mathrm{CH}$ edited and proofread the manuscript. $\mathrm{CL}, \mathrm{ZZ}$, and $\mathrm{MZ}$ supervised the writing process and approved the manuscript. All authors reviewed the final manuscript.
Aspects of Osteoporosis and Osteoarthritis (ESCEO). Aging Clin. Exp. Res. 28 (1), 1-16. doi: 10.1007/s40520-015-0522-1

Cs-Szabo, G., Roughley, P. J., Plaas, A. H., and Glant, T. T. (1995). Large and small proteoglycans of osteoarthritic and rheumatoid articular cartilage. Arthritis Rheumatism 38 (5), 660-668. doi: 10.1002/art.1780380514

Embree, M. C., Kilts, T. M., Ono, M., Inkson, C. A., Syed-Picard, F., Karsdal, M. A., et al. (2010). Biglycan and fibromodulin have essential roles in regulating chondrogenesis and extracellular matrix turnover in temporomandibular joint osteoarthritis. Am. J. Pathol. 176 (2), 812-826. doi: 10.2353/ajpath.2010.090450

Fava, R. A., Olsen, N. J., Postlethwaite, A. E., Broadley, K. N., Davidson, J. M., Nanney, L. B., et al. (1991). Transforming growth factor beta 1 (TGF-beta 1) induced neutrophil recruitment to synovial tissues: implications for TGF-betadriven synovial inflammation and hyperplasia. J. Exp. Med. 173 (5), 1121-1132. doi: 10.1084/jem.173.51121

Gill, M. R., Oldberg, A., and Reinholt, F. P. (2002). Fibromodulin-null murine knee joints display increased incidences of osteoarthritis and alterations in tissue biochemistry. Osteoarthritis Cartilage 10 (10), 751-757. doi: 10.1053/joca.20020527

Greenblatt, M. B., Ritter, S. Y., Wright, J., Tsang, K., Hu, D., Glimcher, L. H., et al. (2013). NFATc1 and NFATc2 repress spontaneous osteoarthritis. Proc. Natl. Acad. Sci. U. S. A. 110 (49), 19914-19919. doi: 10.1073/pnas.1320036110

Habib, G. S., Saliba, W., and Nashashibi, M. (2010). Local effects of intra-articular corticosteroids. Clin. Rheumatol 29 (4), 347-356. doi: 10.1007/s10067-009-1357-y

Hedbom, E., and Heinegard, D. (1989). Interaction of a 59-kDa connective tissue matrix protein with collagen I and collagen II. J. Biol. Chem. 264 (12), 6898-6905.

Hong, J. H., Lee, G. T., Lee, J. H., Kwon, S. J., Park, S. H., Kim, S. J., et al. (2009). Effect of bone morphogenetic protein-6 on macrophages. Immunology 128 (1 Suppl), e442-e450. doi: 10.1111/j.1365-2567.2008.02998.x

Jian, J., Zheng, Z., Zhang, K., Rackohn, T. M., Hsu, C., Levin, A., et al. (2013). Fibromodulin promoted in vitro and in vivo angiogenesis. Biochem. Biophys. Res. Commun. 436 (3), 530-535. doi: 10.1016/j.bbrc.2013.06.005

Kashiwagi, M., Enghild, J. J., Gendron, C., Hughes, C., Caterson, B., Itoh, Y., et al. (2004). Altered proteolytic activities of ADAMTS-4 expressed by C-terminal processing. J. Biol. Chem. 279 (11), 10109-10119. doi: 10.1074/jbc.M312123200

Kuyinu, E. L., Narayanan, G., Nair, L. S., and Laurencin, C. T. (2016). Animal models of osteoarthritis: classification, update, and measurement of outcomes. J. Orthopaedic Surg. Res. 11. doi: 10.1186/s13018-016-0346-5

Lauder, R. M., Huckerby, T. N., and Nieduszynski, I. A. (1996). The structure of the keratan sulphate chains attached to fibromodulin isolated from articular cartilage. Eur. J. Biochem. 242 (2), 402-409. doi: 10.1111/j.1432-1033.1996.0402r.x

Lauder, R. M., Huckerby, T. N., Nieduszynski, I. A., and Plaas, A. H. (1998). Agerelated changes in the structure of the keratan sulphate chains attached to fibromodulin isolated from articular cartilage. Biochem. J. 330 (Pt 2), 753-757. doi: $10.1042 / b j 3300753$ 
Lee, E. J., Jan, A. T., Baig, M. H., Ahmad, K., Malik, A., Rabbani, G., et al. (2018a). Fibromodulin and regulation of the intricate balance between myoblast differentiation to myocytes or adipocyte-like cells. FASEB J. 32 (2), 768-781. doi: 10.1096/f. 201700665 R

Lee, E. J., Nam, J. H., and Choi, I. (2018b). Fibromodulin modulates myoblast differentiation by controlling calcium channel. Biochem. Biophys. Res. Commun. 503 (2), 580-585. doi: 10.1016/j.bbrc.2018.06.041

Li, C., and Zheng, Z. (2018). What's the future of osteoarthritis treatment. Acta Sci. Orthopaedics 1 (3), 01-02.

Li, C. S., Yang, P., Ting, K., Aghaloo, T., Lee, S., Zhang, Y., et al. (2016). Fibromodulin reprogrammed cells: a novel cell source for bone regeneration. Biomaterials 83 , 194-206. doi: 10.1016/j.biomaterials.2016.01.013

Li, C., Zou, M., and Zheng, Z. (2018). Current medication for osteoarthritis. Acta Sci. Orthopaedics 1 (3), 09-12.

Lin, Z., Fitzgerald, J. B., Xu, J., Willers, C., Wood, D., Grodzinsky, A. J., et al. (2008). Gene expression profiles of human chondrocytes during passaged monolayer cultivation. J. Orthopaedic Res. : Off. Publ. Orthopaedic Res. Soc. 26 (9), 12301237. doi: $10.1002 /$ jor.20523

Melching, L. I., and Roughley, P. J. (1999). Modulation of keratan sulfate synthesis on lumican by the action of cytokines on human articular chondrocytes. Matrix Biol. J. Int. Soc. Matrix Biol. 18 (4), 381-390. doi: 10.1016/ S0945-053X(99)00033-5

Melrose, J., Fuller, E. S., Roughley, P. J., Smith, M. M., Kerr, B., Hughes, C. E., et al. (2008). Fragmentation of decorin, biglycan, lumican and keratocan is elevated in degenerate human meniscus, knee and hip articular cartilages compared with age-matched macroscopically normal and control tissues. Arthritis Res. Ther. 10 (4), R79. doi: 10.1186/ar2453

Miclea, R. L., Siebelt, M., Finos, L., Goeman, J. J., Lowik, C. W., Oostdijk, W., et al. (2011). Inhibition of Gsk3beta in cartilage induces osteoarthritic features through activation of the canonical Wnt signaling pathway. Osteoarthritis cartilage 19 (11), 1363-1372. doi: 10.1016/j.joca.2011.07.014

Mitani, Y., Honda, A., and Jasin, H. E. (2001). Polymorphonuclear leukocyte adhesion to articular cartilage is inhibited by cartilage surface macromolecules. Rheumatol. Int. 20 (5), 180-185. doi: 10.1007/s002960000098

Monfort, J., Tardif, G., Reboul, P., Mineau, F., Roughley, P., Pelletier, J. P., et al. (2006). Degradation of small leucine-rich repeat proteoglycans by matrix metalloprotease-13: identification of a new biglycan cleavage site. Arthritis Res. Ther. 8 (1), R26. doi: 10.1186/ar1873

Murphy, J. M., Heinegard, R., McIntosh, A., Sterchi, D., and Barry, F. P. (1999). Distribution of cartilage molecules in the developing mouse joint. Matrix Biol. J. Int. Soc. Matrix Biol. 18 (5), 487-497. doi: 10.1016/S0945-053X(99)00042-6

Noyori, K., and Jasin, H. E. (1994). Inhibition of human fibroblast adhesion by cartilage surface proteoglycans. Arthritis Rheumatism 37 (11), 1656-1663. doi: $10.1002 /$ art. 1780371115

Plaas, A. H., Neame, P. J., Nivens, C. M., and Reiss, L. (1990). Identification of the keratan sulfate attachment sites on bovine fibromodulin. J. Biol. Chem. 265 (33), 20634-20640.

Pourhanifeh, M. H., Mohammadi, R., Noruzi, S., Hosseini, S. A., Fanoudi, S., Mohamadi, Y., et al. (2019). The role of fibromodulin in cancer pathogenesis: implications for diagnosis and therapy. Cancer Cell Int. 19. doi: 10.1186/ s12935-019-0870-6

Roughley, P. J., White, R. J., Cs-Szabo, G., and Mort, J. S. (1996). Changes with age in the structure of fibromodulin in human articular cartilage. Osteoarthritis cartilage 4 (3), 153-161. doi: 10.1016/S1063-4584(96)80011-2

Saamanen, A. M., Salminen, H. J., Rantakokko, A. J., Heinegard, D., and Vuorio, E. I. (2001). Murine fibromodulin: cDNA and genomic structure, and age-related expression and distribution in the knee joint. Biochem. J. 355 (Pt 3), 577-585. doi: $10.1042 /$ bj3550577

Scott, D. L. (2010). "Arthritis in the Elderly," in Brocklehurst's Textbook of Geriatric Medicine and Gerontology. Eds. H. M. Fillit, K. Rockwood, and K. Woodhouse (Phiadelphia: Saunders, Elservier), 566-576. doi: 10.1016/ B978-1-4160-6231-8.10070-4

Shirakura, M., Kram, V., Robinson, J., Sikka, S., Kilts, T. M., Wadhwa, S., et al. (2017). Extracellular matrix mediates BMP-2 in a model of temporomandibular joint osteoarthritis. Cells Tissues Organs 204 (2), 84-92. doi: 10.1159/000464102
Shu, C. C., Flannery, C. R., Little, C. B., and Melrose, J. (2019). Catabolism of Fibromodulin in developmental rudiment and pathologic articular cartilage demonstrates novel roles for MMP-13 and ADAMTS-4 in C-terminal processing of SLRPs. Int. J. Mol. Sci. 20 (3). doi: 10.3390/ijms20030579

Sztrolovics, R., White, R. J., Poole, A. R., Mort, J. S., and Roughley, P. J. (1999). Resistance of small leucine-rich repeat proteoglycans to proteolytic degradation during interleukin-1-stimulated cartilage catabolism. Biochem. J. 339 (Pt 3), 571-577. doi: 10.1042/bj3390571

Taruc-Uy, R. L., and Lynch, S. A. (2013). Diagnosis and treatment of osteoarthritis. Primary Care 40 (4), 821-836. doi: 10.1016/j.pop.2013.08.003

Verbruggen, G., Wittoek, R., Cruyssen, B. V., and Elewaut, D. (2012). Tumour necrosis factor blockade for the treatment of erosive osteoarthritis of the interphalangeal finger joints: a double blind, randomised trial on structure modification. Ann. Rheum Dis. 71 (6), 891-898. doi: 10.1136/ard.2011.149849

Wadhwa, S., Embree, M., Ameye, L., and Young, M. F. (2005a). Mice deficient in biglycan and fibromodulin as a model for temporomandibular joint osteoarthritis. Cells Tissues Organs 181 (3-4), 136-143. doi: 10.1159/000091375

Wadhwa, S., Embree, M. C., Kilts, T., Young, M. F., and Ameye, L. G. (2005b). Accelerated osteoarthritis in the temporomandibular joint of biglycan/ fibromodulin double-deficient mice. Osteoarthritis Cartilage 13 (9), 817-827. doi: 10.1016/j.joca.2005.04.016

Yaykasli, K. O., Oohashi, T., Hirohata, S., Hatipoglu, O. F., Inagawa, K., Demircan, K., et al. (2009). ADAMTS9 activation by interleukin 1 beta via NFATc1 in OUMS27 chondrosarcoma cells and in human chondrocytes. Mol. Cell. Biochem. 323 (1-2), 69-79. doi: 10.1007/s11010-008-9965-4

Zhang, W., Cheng, P., Hu, W., Yin, W., Guo, F., Chen, A., et al. (2018). Downregulated microRNA-340-5p promotes proliferation and inhibits apoptosis of chondrocytes in osteoarthritis mice through inhibiting the extracellular signal-regulated kinase signaling pathway by negatively targeting the FMOD gene. J. Cell. Physiol. 234 (1), 927-939. doi: 10.1002/jcp.26921

Zheng, Z., Jian, J., Zhang, X., Zara, J. N., Yin, W., Chiang, M., et al. (2012). Reprogramming of human fibroblasts into multipotent cells with a single ECM proteoglycan, fibromodulin. Biomaterials 33 (24), 5821-5831. doi: 10.1016/j. biomaterials.2012.04.049

Zheng, Z., Jian, J., Velasco, O., Hsu, C. Y., Zhang, K., Levin, A., et al. (2014). Fibromodulin enhances angiogenesis during cutaneous wound healing. Plast. Reconstructive Surg. Global Open 2 (12), e275. doi: 10.1097/GOX.0000000000000243

Zheng, Z., James, A. W., Li, C., Jiang, W., Wang, J. Z., Chang, G. X., et al. (2017). Fibromodulin reduces scar formation in adult cutaneous wounds by eliciting a fetal-like phenotype. Signal Transduction Targeted Ther. 2. doi: 10.1038/ sigtrans.2017.50

Zheng, Z., Li, C., Ha, P., Chang, G. X., Yang, P., Zhang, X., et al. (2019). CDKN2B upregulation prevents teratoma formation in multipotent fibromodulinreprogrammed cells. J. Clin. Invest. 129 (8), 3236-3251. doi: 10.1172/JCI125015

Conflict of Interest: $\mathrm{ZZ}$ is an inventor on fibromodulin-related patents assigned to UCLA. ZZ is a founder of Scarless Laboratories Inc., which sublicenses fibromodulin-related patents from the UC Regents, who also hold equity in the company. ZZ is also a former officer of Scarless Laboratories Inc.

The handling editor is currently organizing a Research Topic with one of the authors $\mathrm{ZZ}$ and confirms the absence of any other collaboration.

The remaining authors declare that the research was conducted in the absence of any commercial or financial relationships that could be construed as a potential conflict of interest.

Copyright $\odot 2019 \mathrm{Li}$, Ha, Jiang, Haveles, Zheng and Zou. This is an open-access article distributed under the terms of the Creative Commons Attribution License (CC BY). The use, distribution or reproduction in other forums is permitted, provided the original author(s) and the copyright owner(s) are credited and that the original publication in this journal is cited, in accordance with accepted academic practice. No use, distribution or reproduction is permitted which does not comply with these terms. 